\title{
EDITORIAL
}

\section{Cosmic Aesthetics}

\section{Stephen E. Braude}

https://doi.org/10.31275/2020/1755

Creative Commons License CC-BY-NC

In my book Immortal Remains (Braude, 2003),

I considered an intriguing argument William

James offered against the suggestion that mediumistic evidence for postmortem survival could be explained away in normal, or at least

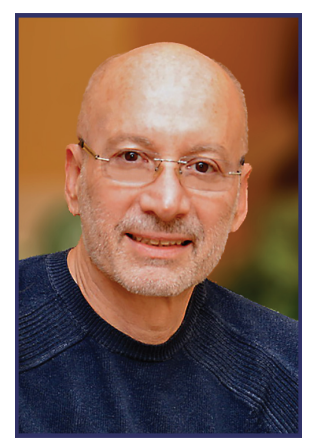
non-survivalist, terms - that is, either by appealing to what I've called The Usual Suspects (e.g., misperception, hidden memories, fraud) or The Unusual Suspects (e.g., dissociation + latent abilities, exceptional memory, or living-agent psi). More specifically, James was concerned with a fascinating, but frustrating, feature of the material gathered from mental mediumship-namely, that even the best cases present a maddening mixture of (a) material suggesting survival, (b) material suggesting psi among the living, and (c) apparent rubbish.

At their best, of course, mediums furnish detailed information for which no normal explanation will suffice. In the cases most strongly suggesting survival, that information concerns the past lives of the deceased. But sometimes mediums also provide information on the present actions, thoughts, and feelings of the living, and that's one reason why some cases suggest psi among the living, and why a livingagent-psi interpretation of mediumship is difficult to rule out. After all, information about present states of affairs is not something to which the deceased would enjoy privileged access.

Moreover, to complicate matters further,

... gems of correct, detailed, and relevant information are nearly always imbedded in an immense matrix of twaddle, 
vagueness, irrelevance, ignorance, pretension, positive error, and occasional prevarication. (Broad, 1962, p. 259)

This mediumistic debris is difficult to interpret, and it's also difficult to ignore. But it's also important not to make too much of it. For one thing, it's easy to imagine why mediums might sometimes (or often) produce communications that are clearly irrelevant, ignorant, vague, etc., even if they get good "hits" on other occasions. For example, we can appeal to analytic overlay, "noise" in the channel, or communicator confusion produced by disembodiment. And for another, there's a large residue of impressive material that clearly can't be explained away in terms of the Usual Suspects (and, arguably, even in terms of some Unusual Suspects) and which can't be simply brushed aside. In fact, assuming that something paranormal is going on, the rubbish might even furnish valuable clues as to the underlying process. Needless to say, any such clues will be welcome; even after more than a century of careful investigation, the nature of mediumship remains largely mysterious. As Broad recognized,

... although instructed opinion is almost unanimous in holding that trance mediumship supplies data which require a paranormal explanation of some kind, there is no consensus of experts in favour of any one suggested paranormal explanation. (Broad, 1962, p. 259)

Of course, there's no reason to suppose that the best cases of mediumship demand only one kind of paranormal explanation. In principle at least, they might exhibit a subtle mixture of psi among the living with manifestations of survival. And as James observed, when we consider the entire spectrum of mediumistic productions from the sublime to the absurd, it's tempting to think that the medium's organism

... not only transmits with great difficulty the influences it receives from beyond the curtain, but mixes its own automatic tendencies most disturbingly therewith. (James, 1909, p. 277) 
Later, James suggests

Extraneous "wills to communicate" may contribute to the results as well as a "will to personate," and the two kinds of will may be distinct in entity, though capable of helping each other out.... The two wills might thus strike up a sort of partnership and stir each other up. It might even be that the "will to personate" would be inert unless it were aroused to activity by the other will. (James, 1909, p. 356)

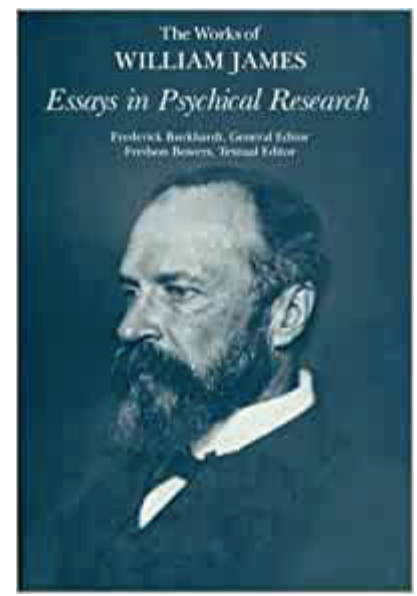

Gauld dubbed this the theory of "overshadowing." As he described it, behind the medium's

... dramatic rendering of communication from the dead, overshadowing it and somehow directing its course, there might sometimes lie those same deceased persons who figure as characters in the drama. The medium writes many of the speeches, and ensures continuity in the plot; but some of the lines (perhaps the most important ones) are filled in by outside authors. (Gauld, 1982, pp. 117-118)

This brings me to the novel argument from James that I want to discuss. He suggested that it may be antecedently incredible that the entire mass of mediumistic communications is nothing but humbug, as it would be if we could explain away all instances of mediumship in terms of the Usual and Unusual Suspects, or even in terms of livingagent psi (LAP). All non-survivalist explanations of mediumship contend that "communications" from the deceased are really constructs by the living designed (consciously or unconsciously) merely to appear to be evidence of survival. The LAP versions of those explanations hold that mediums (and maybe other living persons) subconsciously use their psychic abilities to generate these illusions. Of course, explanations in terms of subconscious deception avoid charging mediums with 
criminal or blatant dishonesty. But according to James, as a general explanatory strategy, that gambit seems to posit an implausible degree of duplicity. He wrote,

The notion that so many men and women, in all other respects honest enough, should have this preposterous monkeying [subliminal] self annexed to their personality seems to me so weird that the spirit-theory immediately takes on a more probable appearance. The spirits, if spirits there be, must indeed work under incredible complications and falsifications, but at least if they are present, some honesty is left in a whole department of the universe which otherwise is run by pure deception. The more I realize the quantitative massiveness of the phenomenon and its complexity, the more incredible it seems to me that in a world all of whose vaster features we are in the habit of considering to be sincere at least, however brutal, this feature should be wholly constituted of insincerity.

(James, 1909, pp. 284-285)

In Immortal Remains, I merely acknowledged this argument as

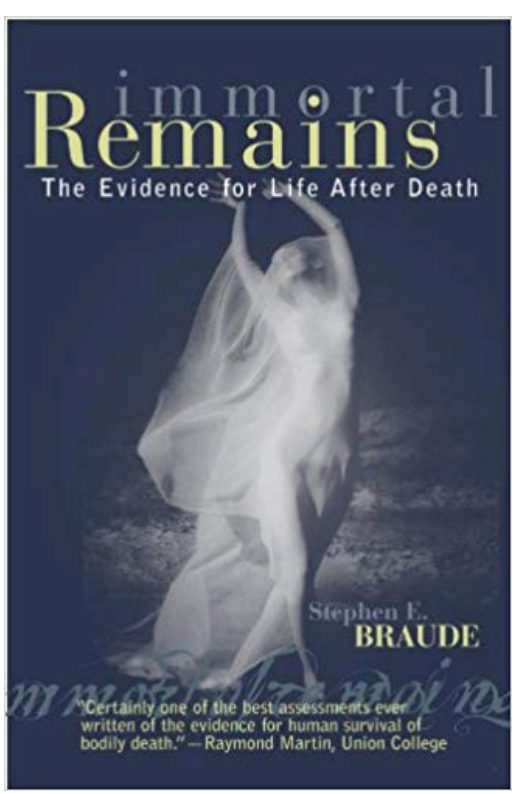
a controversial appeal to cosmic aesthetics, and I noted that it was a strategy about which reasonable and informed people might disagree, or have contrary sensibilities. But now it seems to me that I let James off too easily. James's argument should indeed be considered seriously, but it deserves more commentary than I lavished on it earlier.

Let's first present James's argument somewhat more systematically, to reveal both its structure and its undefended assumptions. I think the following is a fair presentation of that argument: 
(1) If the totality of mediumistic survival evidence can be explained away without positing the survival of personal consciousness, then that body of evidence (a "whole department of the universe") is "run by pure deception."

(2) But it's implausible to suppose that so many otherwise honest men and women would be so thoroughly deceptive in this domain.

(3) Moreover, the "vaster features" of nature, however brutal they may be, are at the very least sincere.

(4) Therefore, it's plausible to suppose that this department of nature is consistent with the rest of nature and is not "wholly constituted of insincerity."

(5) Therefore, the survivalist interpretation of mediumship seems more plausible than the view that the mediumistic evidence can be explained away without positing the survival of personal consciousness.

The first thing to observe about this argument is that it's not logically valid. Rather, it's a form of inference to the best explanation. Moreover, the argument is both vague and contentious in ways that contribute to whatever superficial plausibility it enjoys. Consider, first, James's appeal to a "whole department of the universe" and "vaster features" of nature. To what, exactly, is James referring? How is he parsing the natural world into departments or features?

Domains governed entirely by physical laws (say, plate tectonics or planetary orbits) are neither sincere nor insincere. Indeed, it's a blatant category mistake to assert otherwise. So maybe James had in mind just domains involving living systems. But even that seems too broad; it would also be a category mistake to consider plant photosynthesis to be either sincere or insincere. Indeed, the category of sincerity seems applicable only to living creatures of a certain, and not necessarily very high, level of psychological complexity.

However, once we consider those domains involving living systems in which deception is possible, we find deception throughout nature. Even simple creatures rely on deception to attract and capture prey; many animals feign death in the presence of a predator (because many predators take only live prey); some animals feign injury to 
attract or divert attention away from a mate or from offspring; and some chimps use a kind of verbal deception to mislead other chimps about the location of a food source (see, e.g., Mitchell \& Thompson, 1986). Do these common animal behavioral strategies also count as departments of nature? If so, the mediumistic evidence would not be unprecedented even if it could properly be described as insincere. And needless to say, human deception is a pervasive fact of life in various social situations that equally deserve to be considered departments of nature. Consider courting behavior for example, or political campaigns, or playing poker. So, depending on how finely we choose to parse departments of Nature, why should it be remarkable that the evidence suggesting survival turned out to be largely, if not wholly, constituted of insincerity?

In any case, it's contentious and surprisingly simplistic for James to describe as insincere or deceptive the state of affairs we'd encounter if the mediumistic evidence could all be explained away in non-survivalist terms. There are several matters to consider here.

First, humans are especially complex psychologically, and they have many competing interests and needs or desires, not all of which are conscious. What's deceptive or misleading relative to one set of interests, etc., may be straightforwardly sincere or direct in connection with another. Consider "white lies," for example, told in order to spare someone's feelings. They're deceptive by virtue of being intentional falsehoods; but they can nevertheless be sincere expressions of concern.

Moreover, it's clear that one can be consciously sincere while subconsciously carrying out a contrary agenda. Indeed, it's a familiar fact of life that we often subconsciously subvert the interests and goals we hold consciously. But when conscious and subconscious agendas are at odds, it's once again unacceptably simplistic and misleading to describe a person as either wholly sincere or insincere.

Besides (and perhaps most important), as far as the mediumistic evidence for survival is concerned, mediums and other survivalists can be honestly confused and mistaken about the origin of ostensible postmortem information, and simply not realize how that information could be accounted for in terms of (say) cryptomnesia or living-agent psi. In fact, that undoubtedly happens quite often. But then these would be instances of conceptual naïvete, not deception or insincerity. And that 
would be the case even if all survivalists were honestly misinterpreting and presenting as evidence of survival material unwittingly gained normally or through living-agent psi.

In that case, however, the person who's confused isn't deceiving anyone. Moreover, the evidence itself is also not deceptive; only an agent of some sort can be deceptive or insincere. But then it's misleading and confused for James to suggest that the entire department of Nature (the totality of mediumistic evidence) could be wholly constituted of insincerity or deception. That's merely a very careless way to describe an easily understood state of affairs. Rather (and quite obviously), the data is difficult to interpret. Any errors and confusions are ours, quite understandably, and probably more often than not quite genuine and sincere. Similarly, those who for centuries thought the Earth was flat were not deceived by an insincere Earth or laws of optics. They simply lacked the means for understanding better what experience presented to them.

In some ways, it's surprising that James could have crafted an argument so psychologically glib. After all, he could be an exceptionally keen and sensitive observer of human behavior (as his Varieties of Religious Experience demonstrates so well). But James, somewhat notoriously, wrote so easily and so well ${ }^{1}$ that he was not always as scrupulous about his arguments as some of his philosophical peersfor example, C. S. Peirce. So I suggest we reject James's appeal to cosmic aesthetics and focus instead on more compelling arguments in the debate over survival. There's still plenty to chew on.

\section{NOTE}

${ }^{1}$ Many would say he wrote better than his brother Henry.

\section{REFERENCES}

Braude, S. E. (2003). Immortal remains: The evidence for life after death. Rowman \& Littlefield.

Broad, C. D. (1962). Lectures on psychical research. Routledge \& Kegan Paul. (Reprinted by Routledge, 2011)

Gauld, A. (1982). Mediumship and survival: A century of investigations. Heinemann. James, W. (1909). Report on Mrs. Piper's Hodgson-Control. In F. Burkhardt \& F. 
Editorial

Bowers (Eds.), The works of William James, Vol. 16: Essays in psychical research (pp. 253-360). Harvard University Press.

Mitchell, R. W., \& Thompson, N. S. (Eds.). (1986). Deception: Perspectives on human and nonhuman deceit. SUNY Press. 